\title{
Synthesis and cytotoxic activity of novel metal complexes derived from methyl-3-(4-chlorophenyl)-3-hydroxy-2,2- dimethylpropanoate as potential CDK8 kinase inhibitors
}

Ahmed Aboelmagd ${ }^{1}$, Samir M. El Rayes*1,Mohamed S. Gomaa ${ }^{2}$,Walid Fathalla ${ }^{3}$,Ibrahim A. I. Ali. ${ }^{1}$, Mohamed S. Nafie ${ }^{1}$, Faheem H. Pottoo ${ }^{4}$, Firdos Alam Khan ${ }^{5}$, Mohamed M. Ibrahim ${ }^{6}$

${ }^{1}$ Department of Chemistry, Faculty of Science, Suez Canal University, Ismailia, Egypt.

${ }^{2}$ Department of Pharmaceutical, College of Clinical Pharmacy, ImamAbdulrahman Bin Faisal University, P. O. Box 1982, Dammam 31441, Kingdom of Saudi Arabia.

${ }^{3}$ Department of Physics and Math., Faculty of Engineering, Port-Said University, Port-Said, Egypt.

${ }^{4}$ Department of Pharmacology, College of Clinical Pharmacy, Imam Abdulrahman Bin Faisal University, P. O. Box 1982, Dammam 31441, Kingdom of Saudi Arabia

${ }^{5}$ Department of Stem Cell Research, Institute of Research and Medical consultations (IRMC), Imam Abdulrahman Bin Faisal University, P. O. Box 1982, Dammam 31441, Saudi Arabia

${ }^{6}$ Department of Chemistry, College of Science, Taif University, P.O. Box 11099, Taif 21944, Saudi Arabia. 


\section{Suplimentary S1 FTIR}

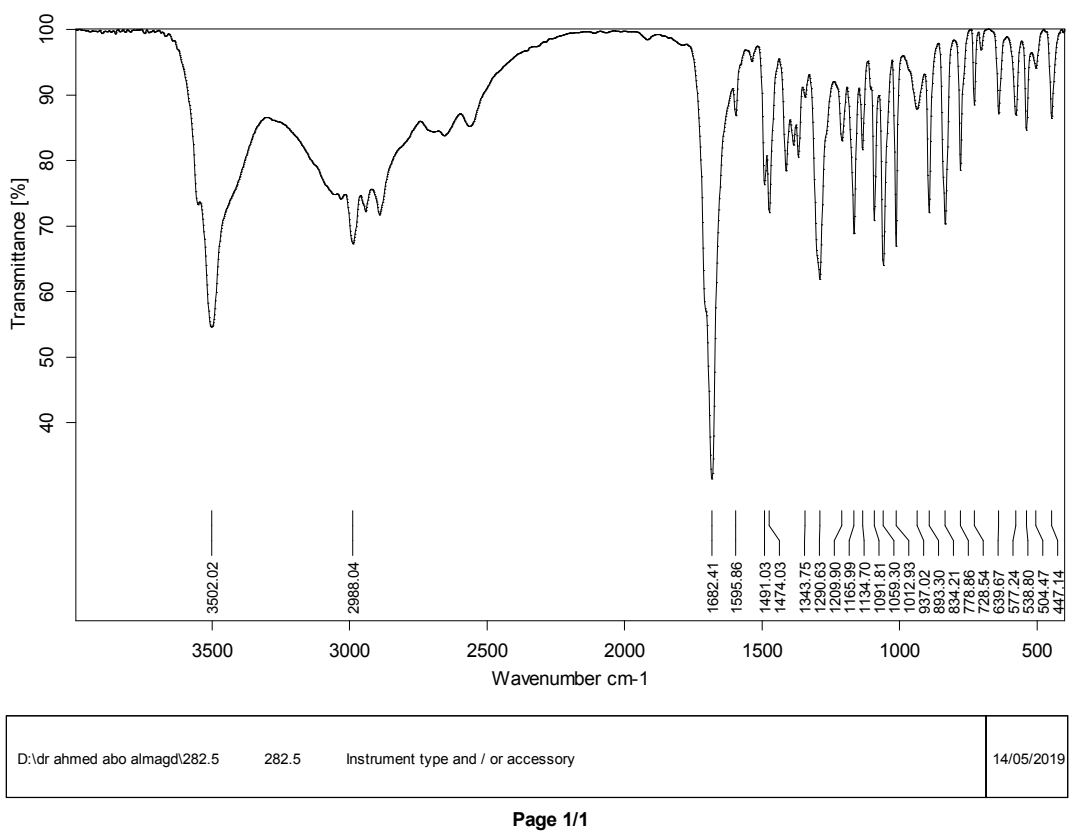

Figure S1: FTIR for methyl-3-(4-chlorophenyl)-3-hydroxy-2,2-

dimethylpropanoic acid (1)

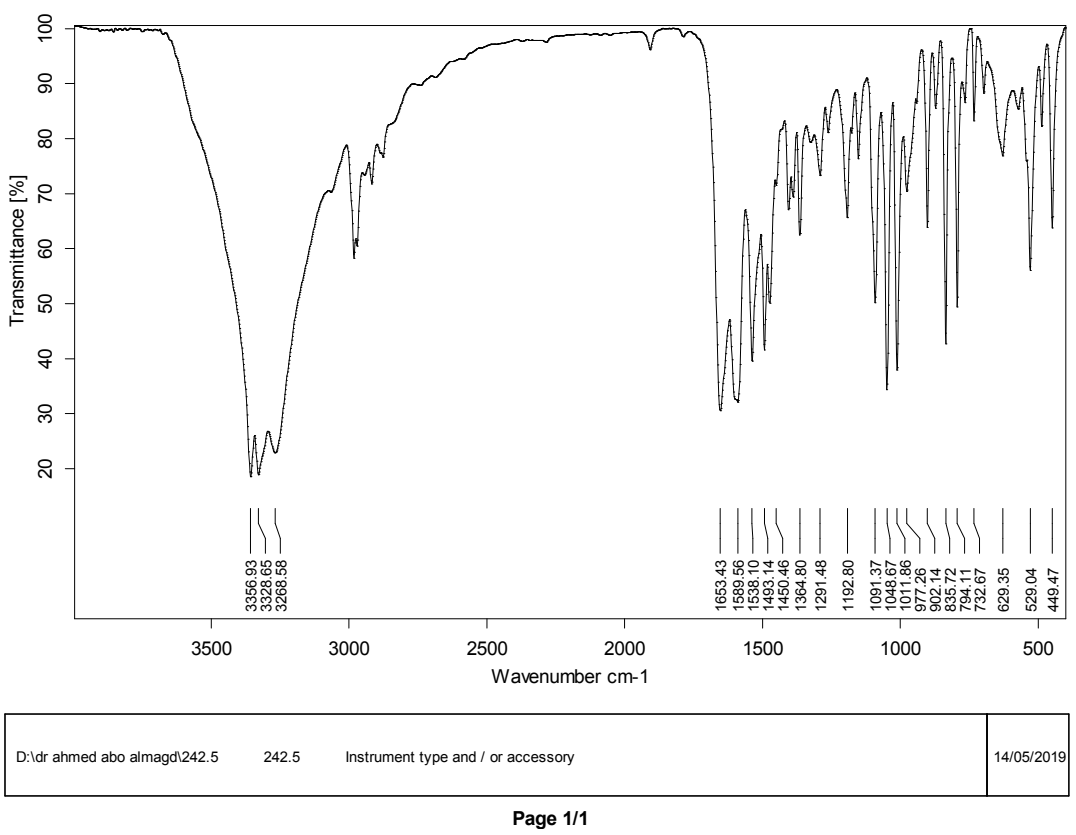

Figure S2: FTIR for methyl-3-(4-chlorophenyl)-3-hydroxy-2,2dimethylpropanoyl hydrazide (2) 


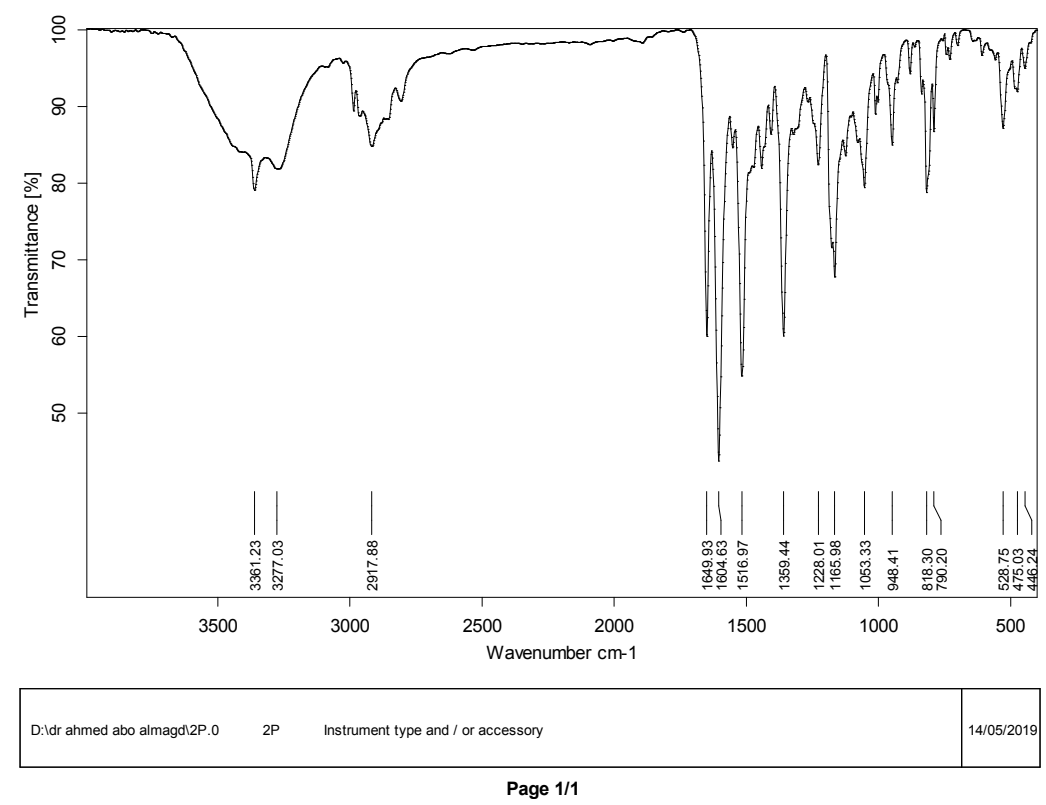

Figure S3: FTIR for methyl-3-(4-chlorophenyl)-3-hydroxy-2,2dimethylpropanoyl hydrazone derivative $\mathbf{3}$

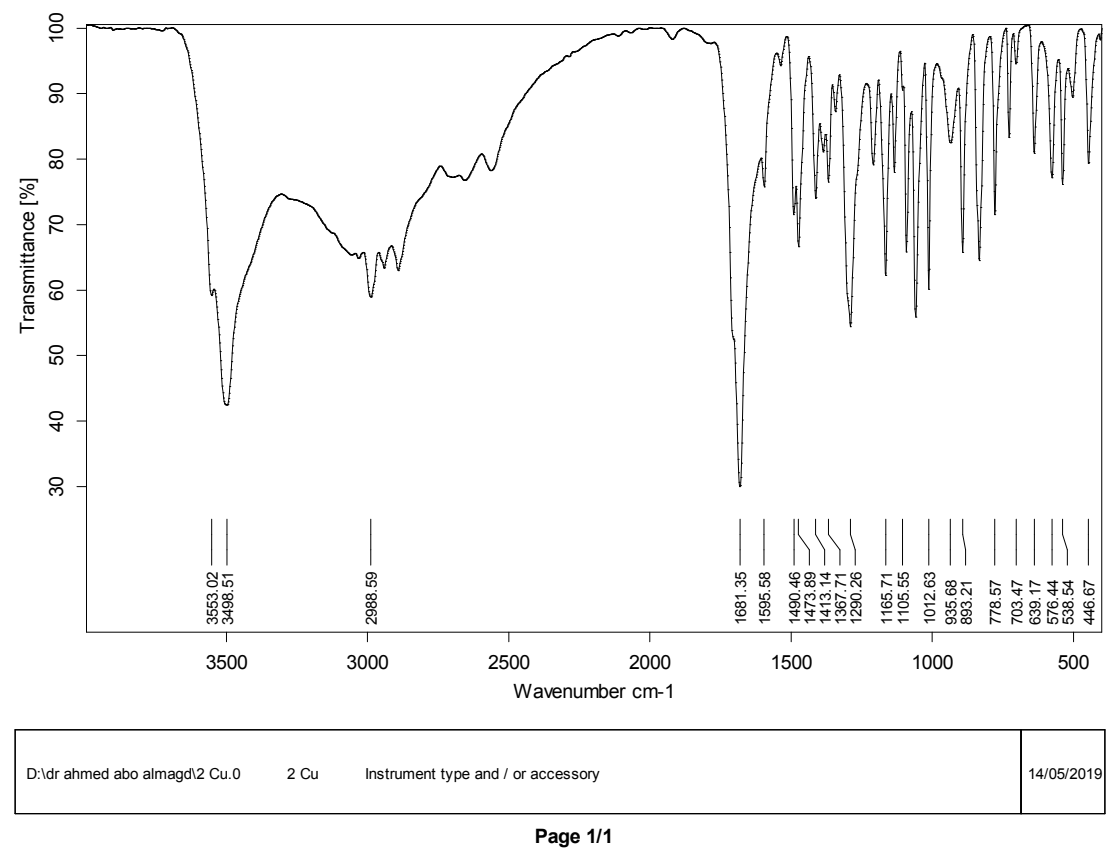

Figure S4: FTIR for copper complex 4a of methyl-3-(4-chlorophenyl)-3hydroxy-2,2-dimethylpropanoic acid 


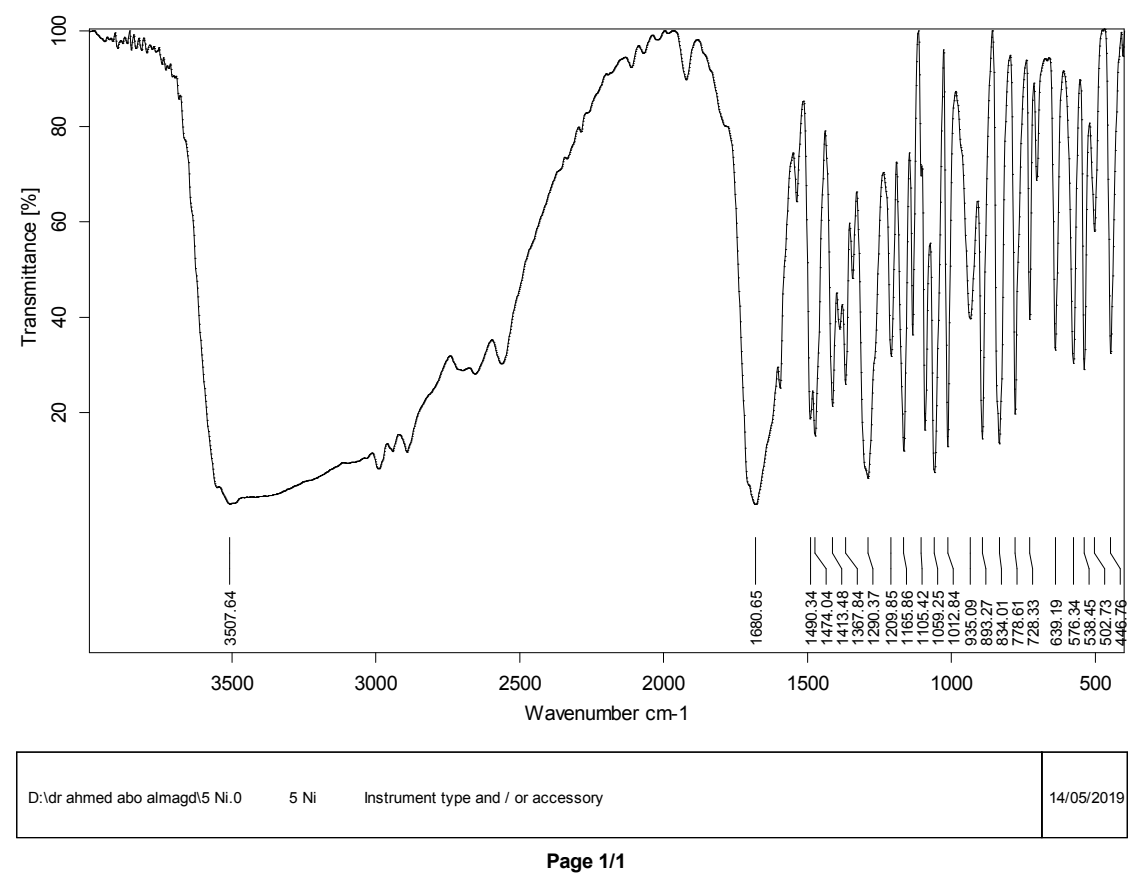

Figure S5: FTIR for nickel complex $\mathbf{4 b}$ of methyl-3-(4-chlorophenyl)-3hydroxy-2,2-dimethylpropanoic acid

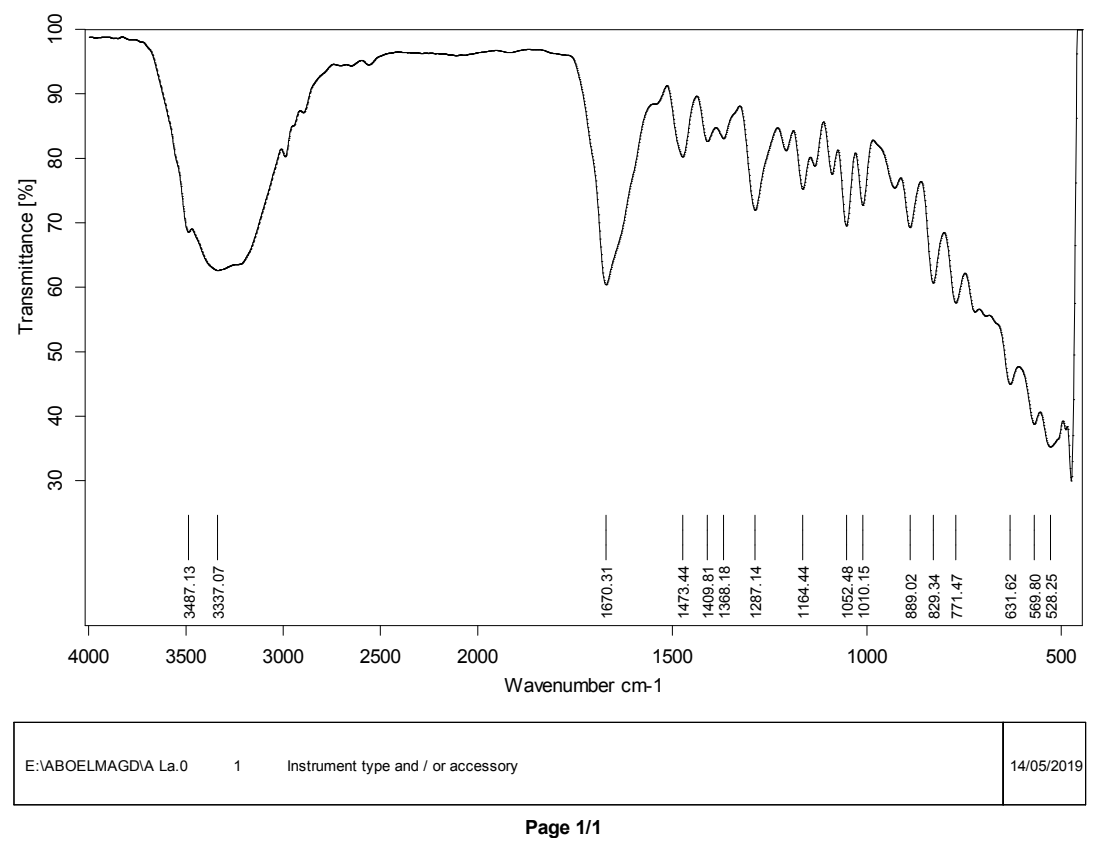

Figure S6: FTIR for lanthanum complex 4c of methyl-3-(4-chlorophenyl)-3hydroxy-2,2-dimethylpropanoic acid 


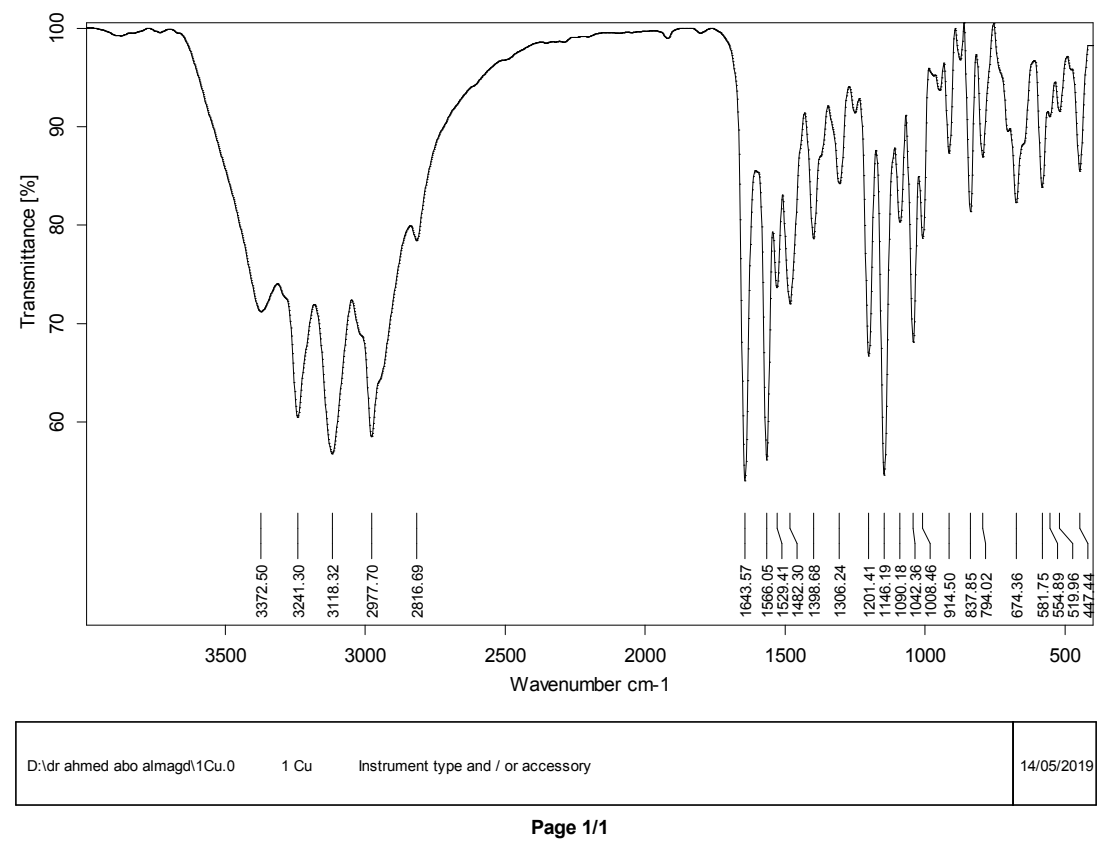

Figure S7: FTIR for copper complex 5a of methyl-3-(4-chlorophenyl)-3hydroxy-2,2-dimethylpropanoyl hydrazide

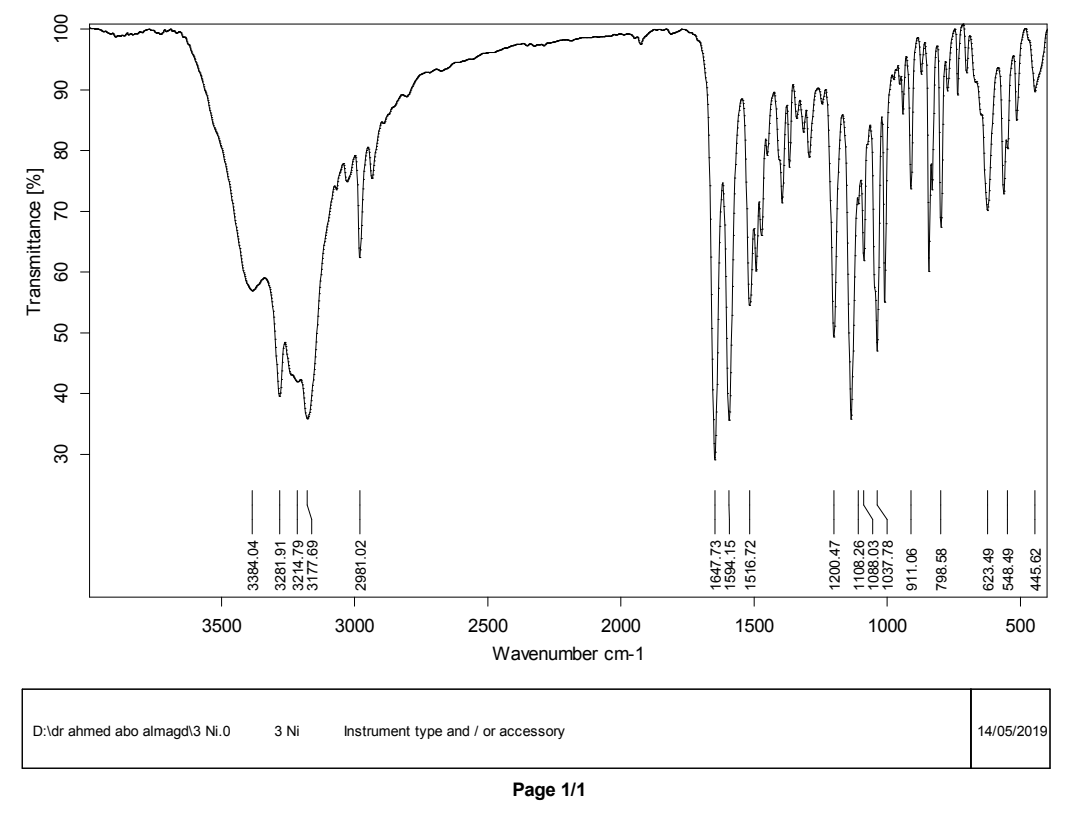

Figure S8: FTIR for nickel complex $\mathbf{5 b}$ of methyl-3-(4-chlorophenyl)-3hydroxy-2,2-dimethylpropanoyl hydrazide 

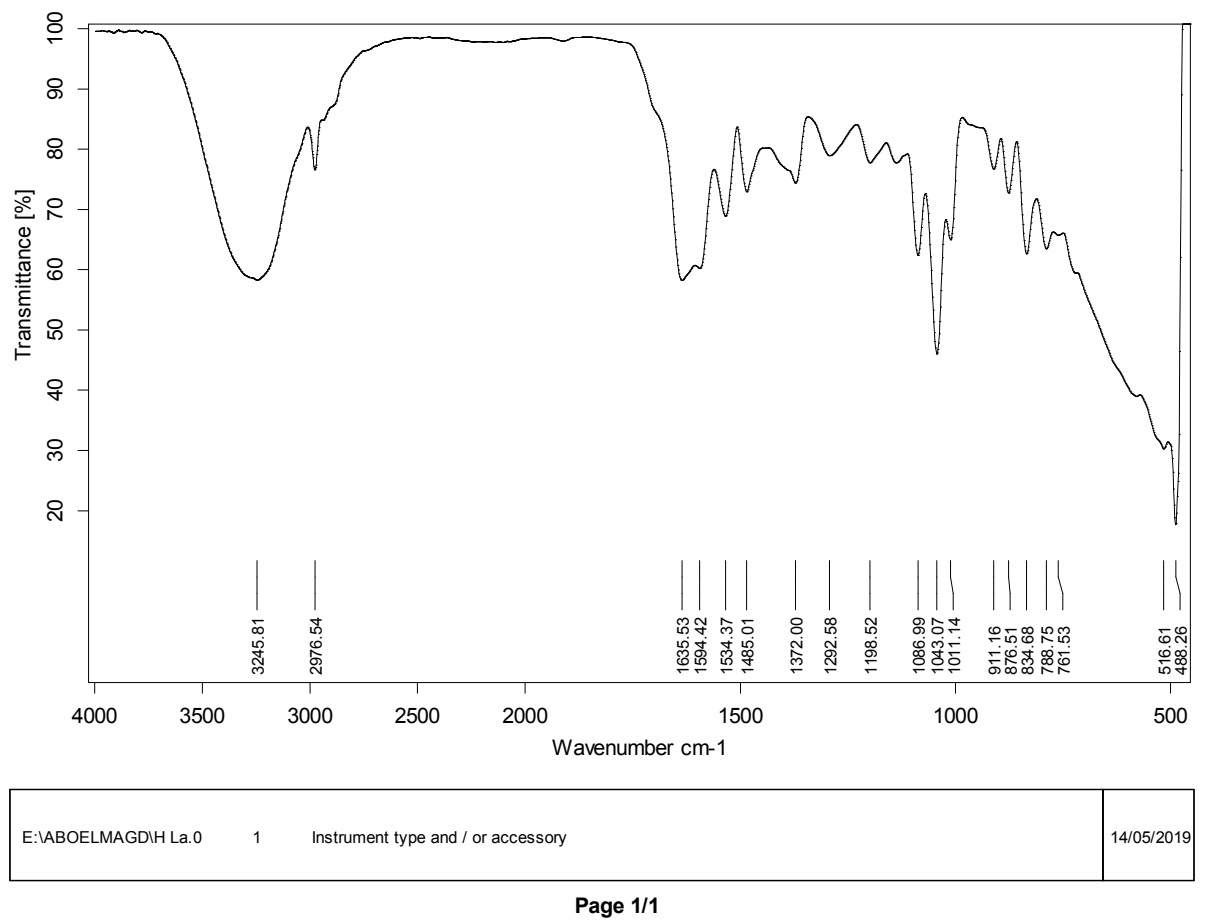

Figure S9: FTIR for lanthanum complex $\mathbf{5 c}$ of methyl-3-(4-chlorophenyl)-3hydroxy-2,2-dimethylpropanoyl hydrazide

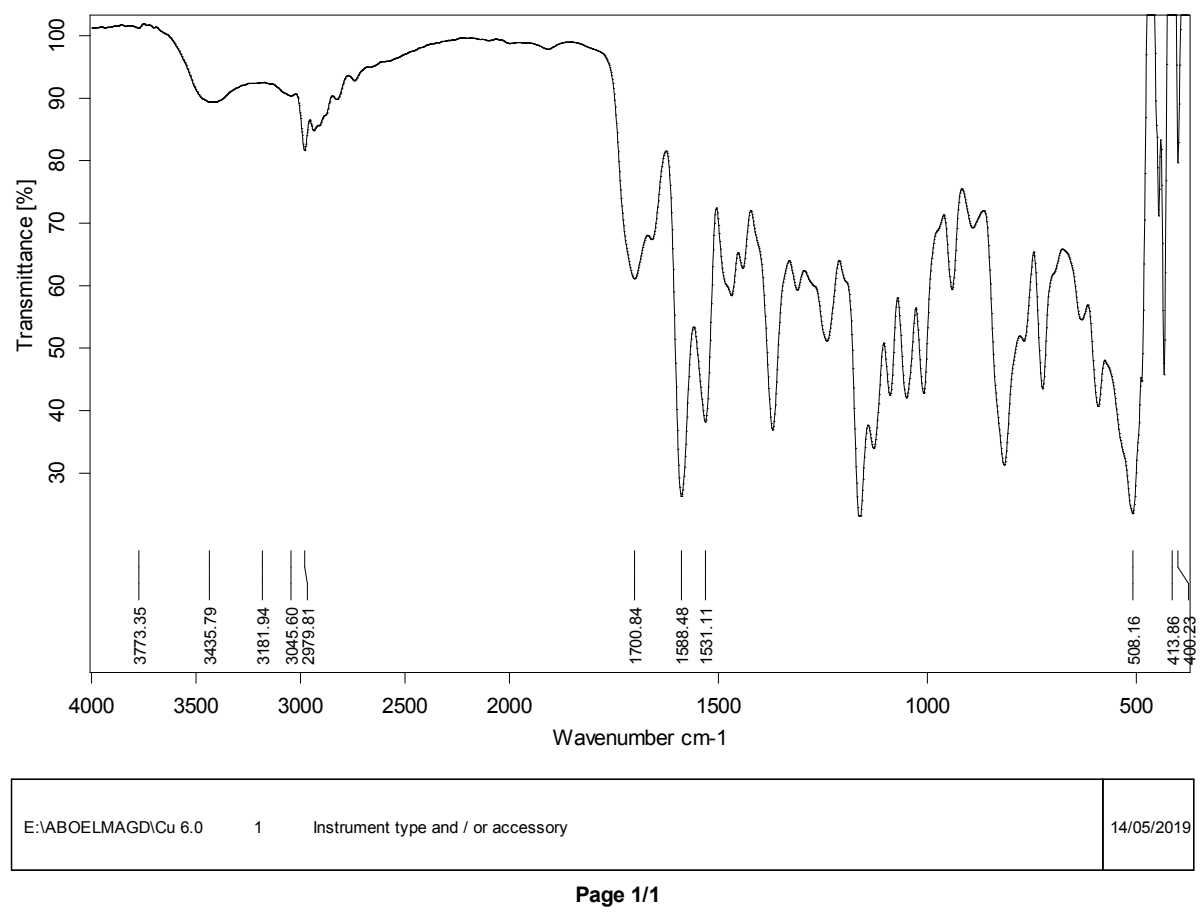

Figure S10: FTIR for copper complex 6a of methyl-3-(4-chlorophenyl)-3hydroxy-2,2-dimethylpropanoyl hydrazone derivative 


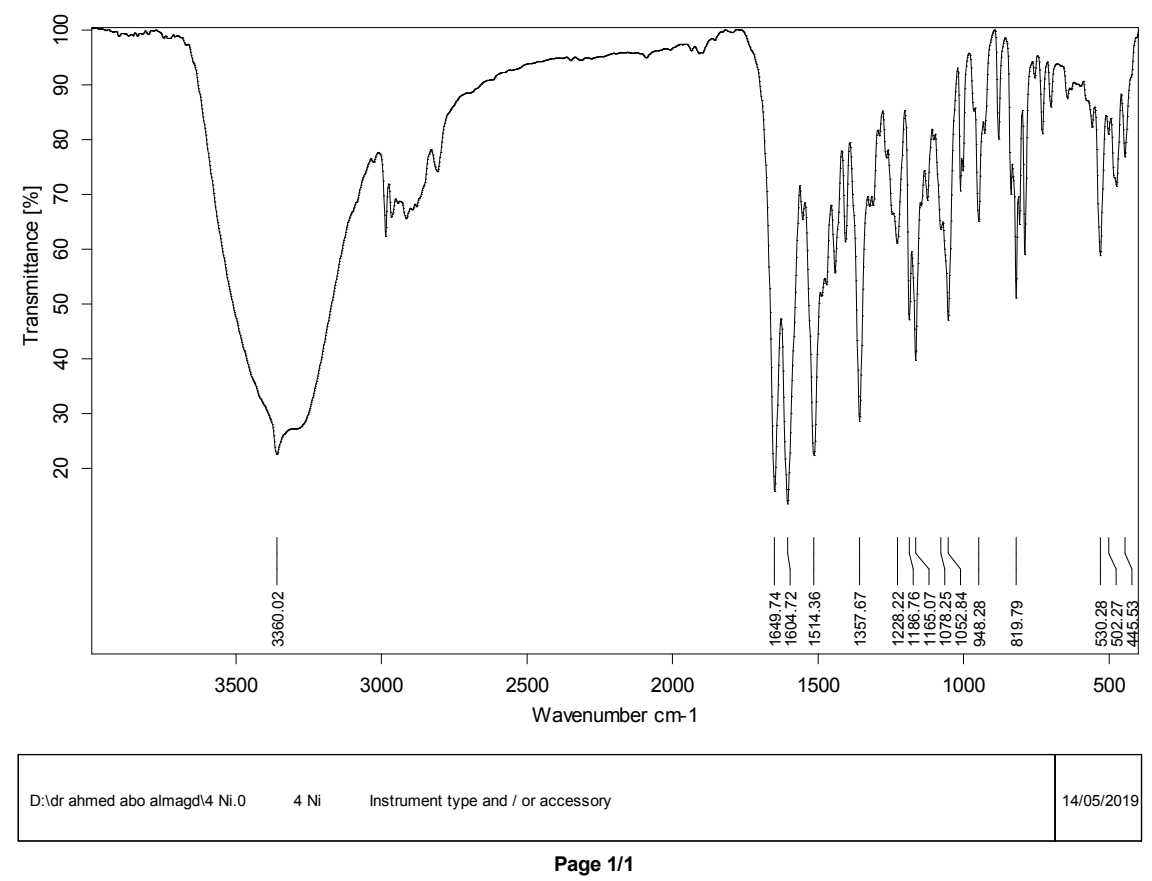

Figure S11: FTIR for nickel complex $\mathbf{6} \mathbf{b}$ of methyl-3-(4-chlorophenyl)-3hydroxy-2,2-dimethylpropanoyl hydrazone derivative

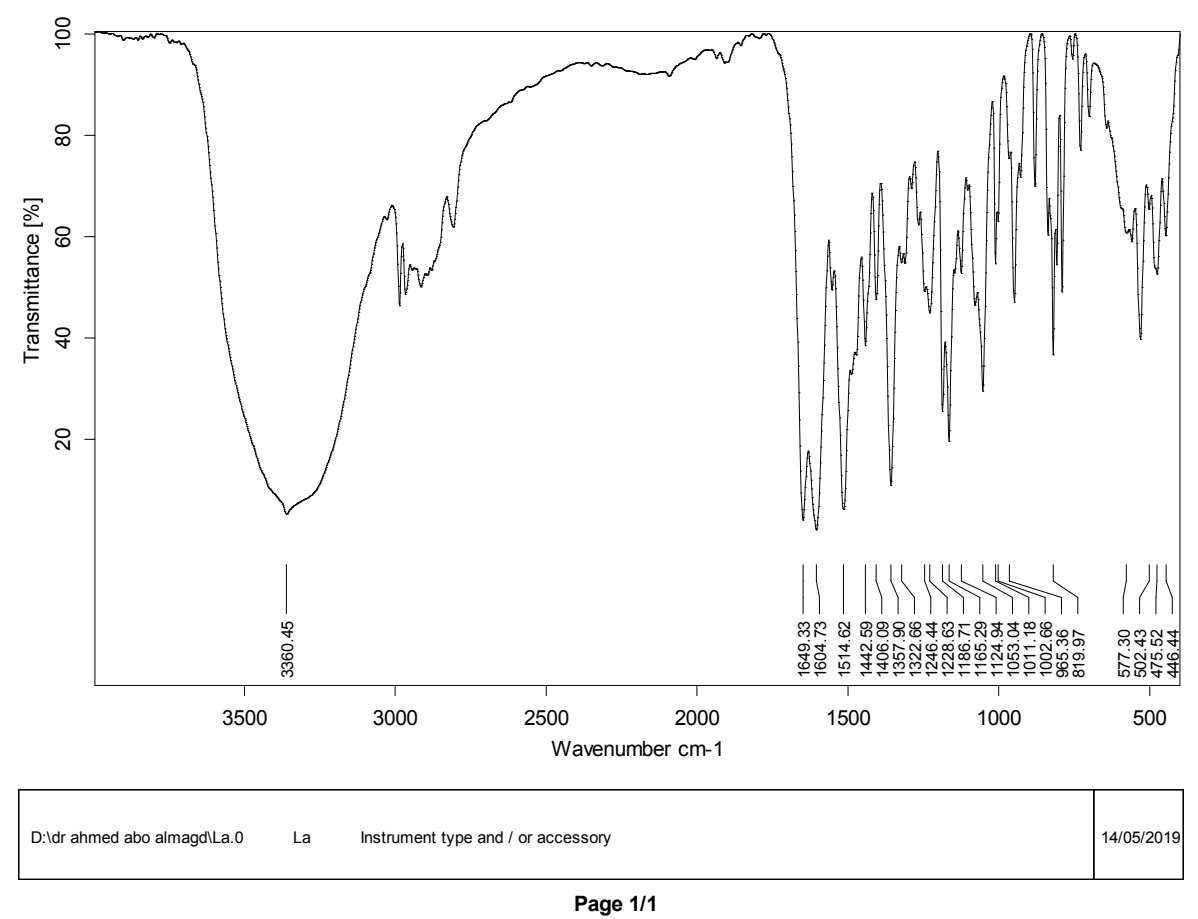

Figure S12: FTIR for lanthanum complex $\mathbf{6 c}$ of methyl-3-(4-chlorophenyl)-3hydroxy-2,2-dimethylpropanoyl hydrazone derivative 
Suplimentary S2 TGA and DTA

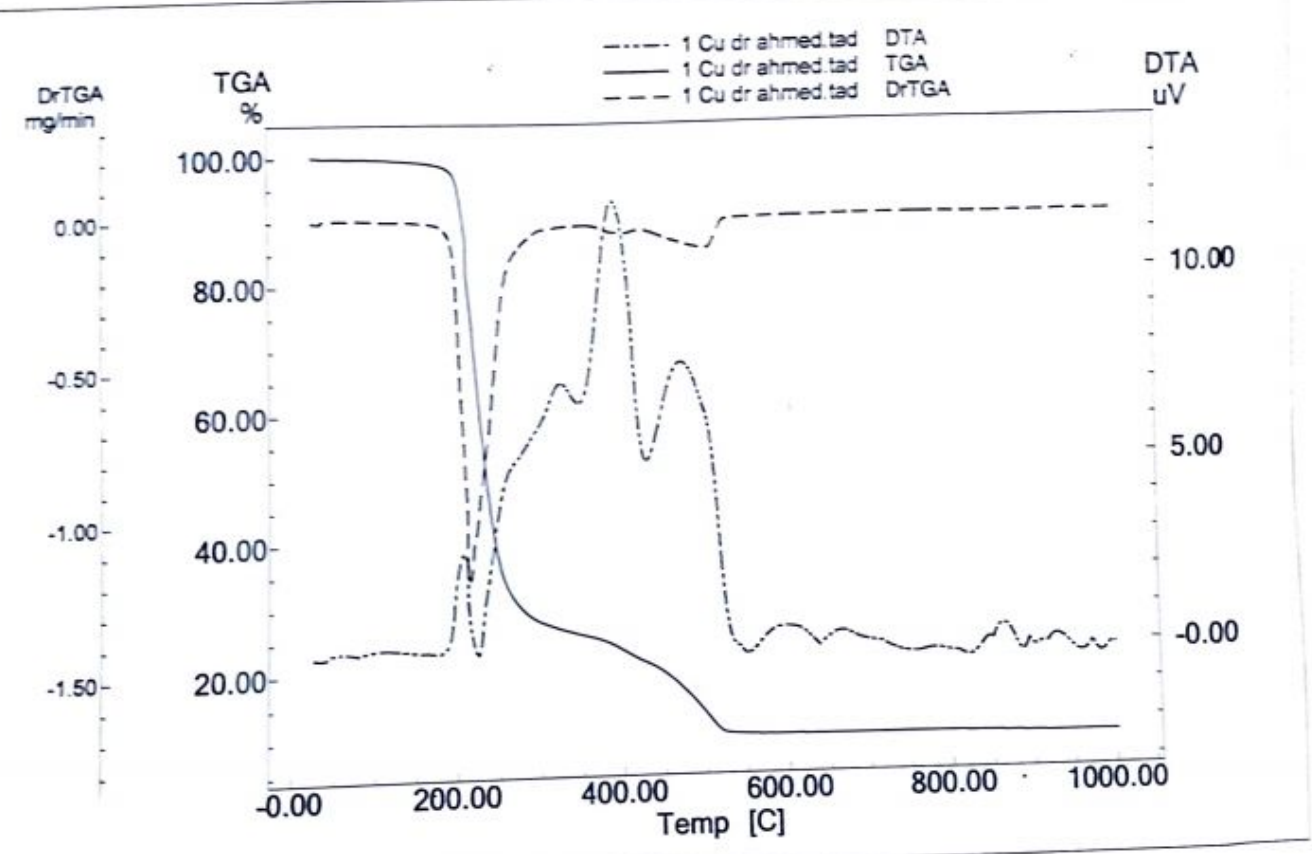

Figure S13: TGA and DTA for copper complex 4a of methyl-3-(4chlorophenyl)-3-hydroxy-2,2-dimethylpropanoic acid

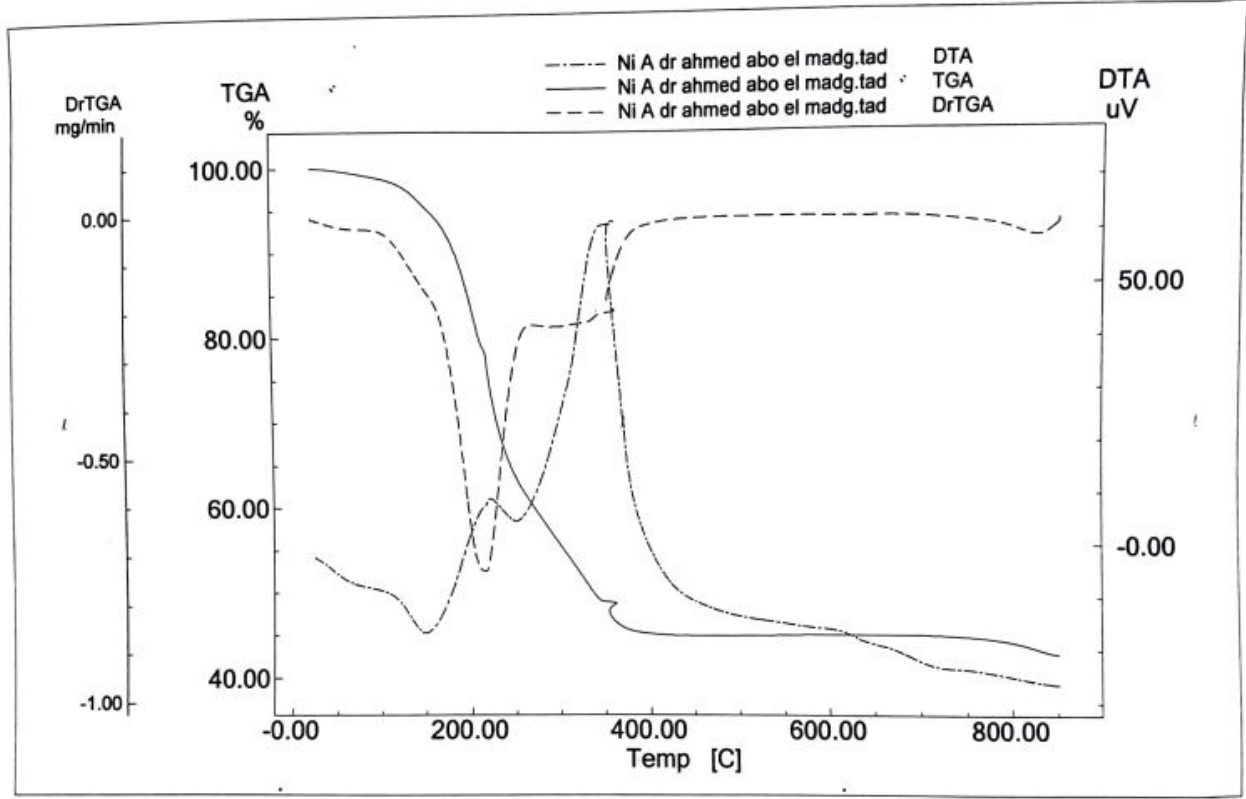

Figure S14: TGA and DTA for nickel complex $\mathbf{4 b}$ of methyl-3-(4chlorophenyl)-3-hydroxy-2,2-dimethylpropanoic acid 


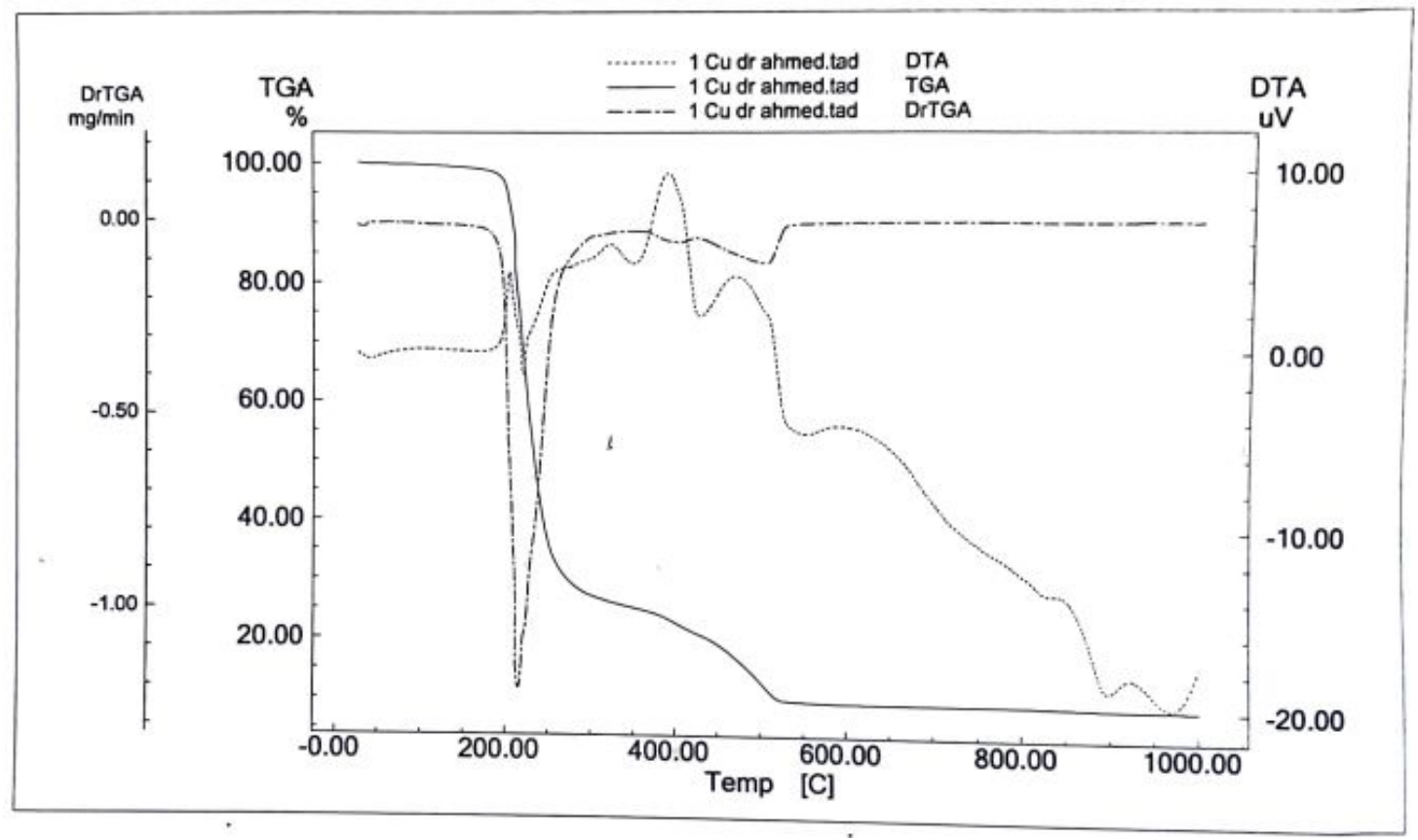

Figure S15: TGA and DTA for copper complex 5a of methyl-3-(4chlorophenyl)-3-hydroxy-2,2-dimethylpropanoyl hydrazide

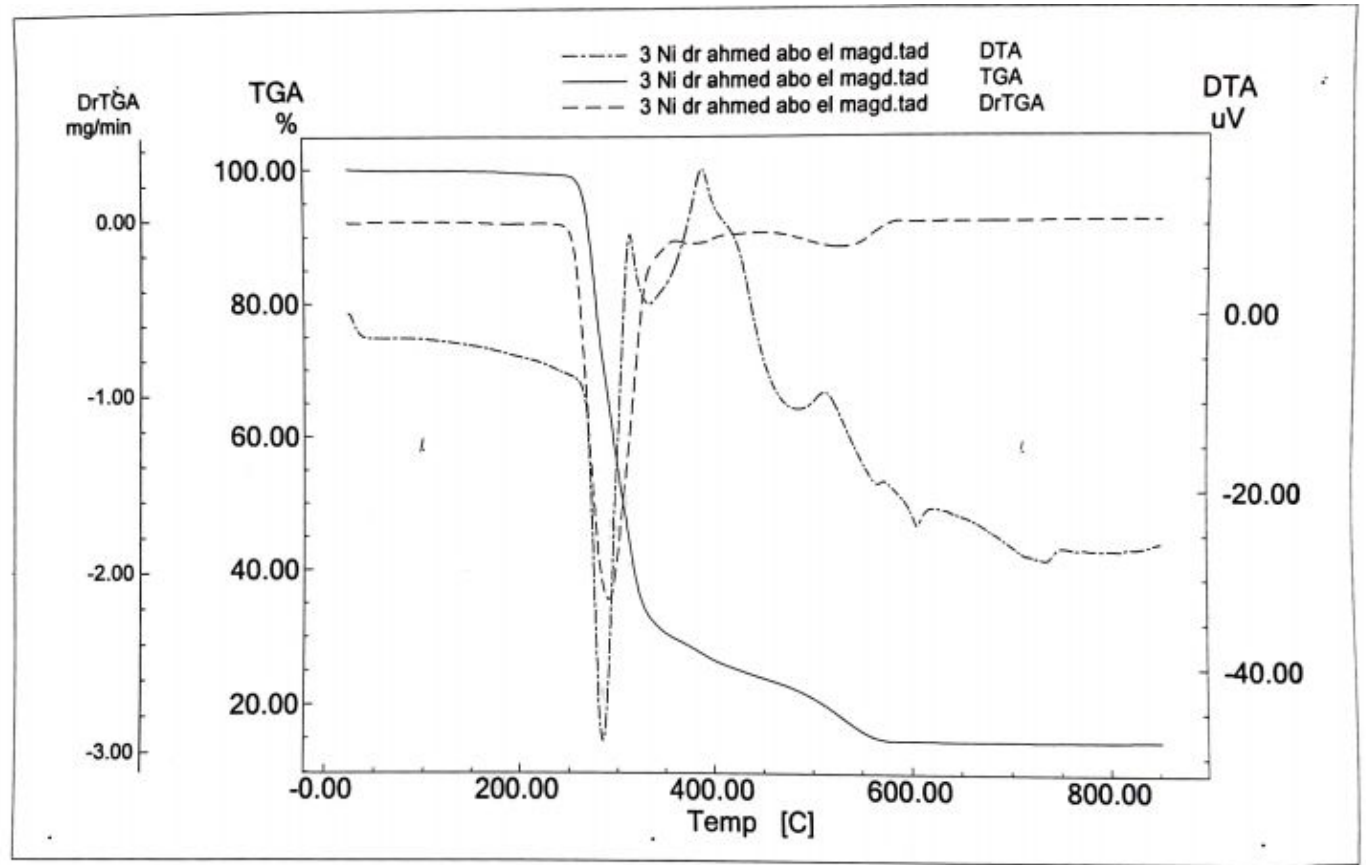

Figure S16: TGA and DTA for nickel complex $\mathbf{5 b}$ of methyl-3-(4chlorophenyl)-3-hydroxy-2,2-dimethylpropanoyl hydrazide 


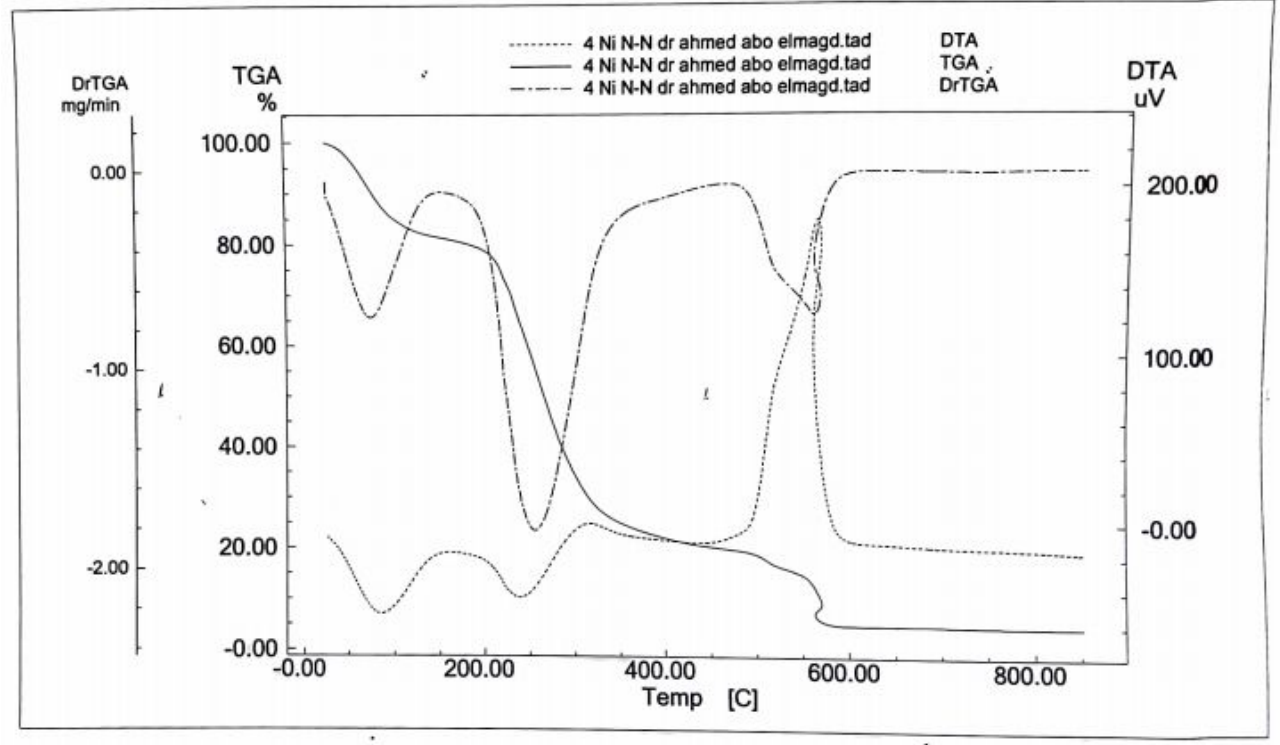

Figure S17: TGA and DTA for nickel complex $\mathbf{6 b}$ of methyl-3-(4chlorophenyl)-3-hydroxy-2,2-dimethylpropanoyl hydrazone derivative 